\title{
Comparação do teste modificado de fixação preferencial com o convencional em pacientes com estrabismo
}

\author{
Comparison of the modified fixation preference test with the conventional test \\ in strabismic patients
}

Edson Procianoy ${ }^{1}$, Letícia Procianoy 2

\section{RESUMO}

Objetivo: Comparara acurácia do teste de fixação preferencial quando o paciente olha e toca o objeto alvo ao invés de apenas olhá-lo, como convencionalmente descrito. Métodos: Estudo piloto transversal e prospectivo, incluindo 40 pacientes estrábicos, com desvios maiores que 10 dioptrias prismáticas entre 7 e 30 anos.

Resultados: O teste modificado mostrou sensibilidade de 93\% (IC 95\%=68,53 $-98,73 \%$ ) e especificidade de $77 \%$ (IC 95\%=57,95-88,97\%). O teste convencional apresentou sensibilidade de 93\% (IC 95\%=68,53 - 98,73\%) e especificidade 46\% (IC 95\%= $28,76-64,54 \%)$

Conclusões: Estes resultados sugerem que a modificação no teste de fixação preferencial, solicitando que o paciente toque o objeto alvo, possa reduzir os resultados falsos positivos do teste.

Descritores: Ambliopia; Acuidade visual; Estrabismo/diagnóstico; Fixação ocular/ fisiologia; Valor preditivo dos testes; Fatores de risco; Testes visuais; Sensibilidade e especificidade; Estudo comparativo

\begin{abstract}
Purpose: To compare the accuracy of the fixation preference test performed with the patient touching and looking at the fixation target, to the conventional method test done with the patient only looking at it.

Methods: A pilot transversal and prospective study was done with 40 strabismic patients with deviations greater than 10 prism diopters, from 7 to 30 years old.

Results: The modified test had a sensitivity of $93 \%$ (IC 95\%=68.53-98.73\%) and specificity of $77 \%$ (IC $95 \%=57.95-88.97 \%$ ); while the conventional test had a sensitivity of $93 \%$ (IC $95 \%=68.53-98.73 \%$ ) and a specificity of $46 \%$ (IC $95 \%=28.76-64.54 \%)$.

Conclusions: Our results suggest that the modification of the fixation preference test by asking the patient to touch the fixation target might reduce the number of false positive results of the test.
\end{abstract}

Keywords: Amblyopia; Visual acuity; Strabismus/diagnosis; Fixation, ocular/physiology; Predictive value of tests; Risk factors; Vision tests; Sensitivity and specificity; Comparative study

\section{INTRODUÇÃO}

Ambliopia é a diminuição da acuidade visual unilateral (o mais frequente) ou bilateral, decorrente de deprivação visual e/ou interação binocular anormal. Não tem causa orgânica detectável ao exame físico do olho, ocorrendo no período de imaturidade do sistema visual(1). Essa é a causa mais comum de baixa acuidade visual na infância (prevalência de 1,6\% a 3,6\%) (2-8). Estrabismo e anisometropia são as causas mais frequentes de ambliopia,ocorrendo em 2 a $5 \%$ da população geral ${ }^{(6-8)}$.

Sabe-se que, devido à maior plasticidade sensorial, quanto mais precoce o tratamento, mais rápida e eficaz será a recuperação visual do paciente. Assim, o diagnóstico precoce e acurado é fundamental para a recuperação visual(2).

No período verbal, o padrão ouro para o diagnóstico de ambliopia é a acuidade visual medida na tabela do ETDRS (Early Treatment Diabetic Retinopathy Study) $)^{(9)}$. Considera-se ambliopia monocular a diferença de visão entre os olhos de duas ou mais linhas. No entanto, para as crianças na fase pré-verbal, o teste de fixação preferencial é o mais utilizado ${ }^{(10)}$.

O teste de fixação preferencial compara a capacidade de fixação de um olho em relação ao outro quando os dois estão abertos. $O$ teste considera que amblíopes monoculares vão apresentar preferência de fixação por um olho e incapacidade de manter fixação com o outro, enquanto que os não amblíopes tenderão a alternar a fixação ou manter melhor a fixação com o olho não preferencial|(11).

Baseado nisso, quando o paciente é incapaz de alternar a fixação espontaneamente, oclui-se momentaneamente o olho dominante para posterior avaliação da capacidade do olho contralateral em manter a fixação no alvo exposto. A maioria dos autores considera não haver ambliopia quando a fixação é mantida por 5 segundos ou mais ${ }^{(12-16)}$.

Os estudos atuais têm mostrado controvérsias quanto à acurácia do teste de fixação preferencial(10). Tendo em vista a importância do diagnóstico e do tratamento na fase pré-verbal é fundamental que se tenha um teste com máxima acurácia. Observamos, na clínica, uma aparente redução dos resultados falsos positivos quando o teste de fixação preferencial era feito com o paciente tocando o objeto alvo.

Assim, o objetivo desse estudo é verificar se a acurácia do teste de fixação preferencial melhora quando o paciente olha e toca o objeto alvo ao invés de apenas olhá-lo, como o teste é classicamente descrito.

\section{MÉTODOS}

Realizou-se um estudo piloto transversal prospectivo em 40 pacientes. Por amostragem sequencial consecutiva, foram incluídos no sidade Federal do Rio Grande do Sul.

Médico, Chefe do Setor de Estrabismo do Hospital de Clínicas de Porto Alegre, Universidade Federa

Médica, Hospital de Clínicas de Porto Alegre - Porto Alegre (RS), Brasil.

Financiamento: Não houve financiamento para este trabalho.

Divulgação de potenciais conflitos de interesse: E.Procianoy, Não; L.Procianoy, Não.

Endereço para correspondência: Edson Procianoy. Av. Carlos Gomes, 1.200 - Cj. 805 - Porto Alegre (RS) - 90480-001 - E-mail: eprocianoy@terra.com.br 
Tabela 1. Características basais dos pacientes

\begin{tabular}{lcc}
\hline & $\begin{array}{c}\text { Amblíopes } \\
\mathbf{N}=\mathbf{1 4}\end{array}$ & $\begin{array}{c}\text { Não amblíopes } \\
\mathbf{N = \mathbf { 2 6 }}\end{array}$ \\
\hline Gênero masculino & $7(50)$ & $14(53,8)$ \\
Idade (anos) & $11,9 \pm 5,8$ & $9,8 \pm 4,7$ \\
Equivalente esférico (D) & $0,5 \pm 2,0$ & $0,7 \pm 2,9$ \\
Tamanho do desvio (DP) & $26,9 \pm 9,1$ & $24,9 \pm 9,5$ \\
Tipo de desvio: & $9(64,2)$ & $17(65,3)$ \\
ET & $5(35,7)$ & $8(30,7)$ \\
XT & $0(0)$ & $1(3,8)$ \\
HT & $0,55 \pm 0,23$ & 0,0 \\
Diferença de acuidade visual & & \\
entre os olhos (logMAR) & & \\
\hline
\end{tabular}

* = dados descritos em $n$ (\%), ou média \pm desvio padrão

estudo pacientes de ambos os sexos, com estrabismo de tamanho >10 DP, entre 7 e 30 anos, que consultaram o Serviço de Oftalmologia do Hospital de Clínicas de Porto Alegre (HCPA). Foram excluídos os pacientes com estrabismo paralítico, com outras causas de baixa acuidade visual que não apenas a ambliopia, e os que não souberam informar a acuidade visual com a tabela do ETDRS. As características basais dos pacientes estão na tabela 1.

O teste do padrão de fixação preferencial em que o paciente apenas olha o objeto alvo é citado aqui como teste convencional, e aquele no qual o paciente é orientado a também tocar o objeto é chamado de teste modificado.

Os dois testes foram realizados em todos os pacientes, em sequência aleatória, pelo mesmo examinador, mascarado quanto à acuidade visual dos pacientes. O alvo de fixação foi a ponta de uma caneta colocada a $37 \mathrm{~cm}$ do paciente. Na realização do teste modificado, solicitava-se que o paciente mantivesse o dedo encostado na ponta da caneta. Pacientes capazes de manter a fixação com o olho não dominante 5 segundos ou mais foram considerados não amblíopes. Aqueles que não mantinham a fixação por esse tempo foram considerados amblíopes.

A medida da acuidade visual foi realizada por um segundo examinador, sempre na mesma sala, com a mesma luminosidade em uma tabela ETDRS. O paciente tinha o olho ocluído pelo examinador com uma ficha de papel a fim de impedir a má oclusão e o embaralhamento visual causado pela possível compressão do globo ocular na oclusão manual. O efeito de "crowding" não foi inibido, sendo mostradas várias linhas simultaneamente, e verificada a acuidade visual desde os maiores optotipos até os menores, seguindo-se as linhas. A acuidade visual registrada foi a da linha em que o paciente leu mais da metade dos optotipos. Para evitar memorização das letras, a acuidade visual do olho não dominante foi sempre testada primeiro. A acuidade visual dos pacientes foi confirmada com o estenopeico a fim de garantir que estivessem usando a melhor correção óptica.

Foram verificadas as proporções de respostas falsas e verdadeiras com os dois métodos e calculou-se a sensibilidade e especificidade de cada teste. Considerou-se ambliopia a diferença de acuidade visual entre os olhos de duas ou mais linhas.

\section{RESULTADOS}

Foram incluídos 40 pacientes, sendo 14 amblíopes. Apenas um desses pacientes não foi diagnosticado como amblíope pelos testes convencional e modificado. Entre os 26 não amblíopes, o teste convencional diagnosticou ambliopia erroneamente (falsos positivos) em 14 pacientes, e o modificado em 6 .

O teste modificado mostrou sensibilidade de 93\% (IC 95\%= $68,53 \%-98,73 \%$ ) e especificidade de $77 \%$ (IC 95\%=57,95\% - 88,97\%). O teste convencional apresentou sensibilidade de 93\% (IC 95\%= $68,53 \%$ - 98,73\%) e especificidade 46\% (IC 95\%=28,76\% - 64,54\%).

\section{DISCUSSÃO}

Verificando-se as sensibilidades e especificidades dos trabalhos publicados, empregando o teste convencional(11-21), com exceção do trabalho de Procianoy, Procianoy ${ }^{(21)}$, todos os demais mostraram especificidade menor do que a sensibilidade (a média das sensibilidades publicadas é de $77,8 \%$ e das especificidades 58\%). Em nosso estudo o teste modificado apresentou especificidade 19\% maior que a média encontrada na literatura. Estes resultados corroboram a idéia de que a modificação no teste de fixação preferencial pode reduzir os resultados falsos positivos. É possível que a participação do paciente ao tocar o alvo desperte o reflexo proprioceptivo e mais atenção(22).

Por tratar-se de um estudo piloto, não podemos concluir que o teste modificado seja mais acurado do que o convencional; porém, estes resultados fortalecem a hipótese de que teremos menos diagnósticos falsos positivos com o seu emprego.

\section{REFERÊNCIAS}

1. Wright KW, Spiegel PH. Pediatric ophthalmology and strabismus. 2 ed. New York Springer; 2003. 1084p

2. Pediatric Eye Disease Investigator Group. The clinical profile of moderate amblyopia in children younger than 7 years. Arch Ophthalmol. 2002;120(3):281-7.

3. Pediatric Eye Disease Investigator Group. A randomized trial of atropine vs. patching for treatment of moderate amblyopia in children. Arch Ophthalmol. 2002; 120(3):268-78. Comment in: JAMA. 2002;287(16):2145-6. Arch Ophthalmol. 2002;120(3):387-8.

4. Eibschitz-Tsimhoni M, Friedman T, Naor J, Eibschitz N, Friedman Z. Early screening for amblyogenic risk factors lowers the prevalence and severity of amblyopia. J AAPOS. 2000;4(4):194-9.

5. Hillis A, Flynn JT, Hawkins BS. The evolving concept of amblyopia: a challenge to epidemiologists. Am J Epidemiol. 1983;118(2):192-205.

6. Gilbert CE, Ellwein LB. Refractive Error Study in Children Study Group. Prevalence and causes of functional low vision in school-age children: results from standardized population surveys in Asia, Africa, and Latin America. Invest Ophthalmol Vis Sci. 2008;49(3): 877-81.

7. Robael D. Rose K, Ojaimi E, Kifley A, Huynh S, Mitchell P. Visual acuity and the causes of visual loss in a population-based sample of 6-year-old Australian children. Ophthalmology. 2005;112(7):1275-82.

8. Williams C, Northstone K, Howard M, Harvey I, Harrad RA, Sparrow JM. Prevalence and risk factors for common vision problems in children: data from the ALSPAC study. $\mathrm{Br}$ J Ophthalmol. 2008;92(7):959-64.

9. Early Treatment Diabetic Retinopathy Study design and baseline patient characteristics. ETDRS report number 7. Ophthalmology. 1991;98(5 Suppl):741-56.

10. American Academy of Ophthalmolgy. Amblyopia. Preferred Practice Pattern ${ }^{\oplus}=$ Guidelines[Internet]. New York: AAO; 2007 [cited 2008 june 1]. Available from: http:// www.aao.org/ppp

11. Knapp P, Moore S. Diagnostic procedures in an orthoptic evaluation. Am Orthopt J. 1962;12:63-9.

12. Sener EC, Mocan MC, Gedik S, Ergin A, Sanaç AS. The reliability of grading the fixation preference test for the assessment of interocular visual acuity differences in patients with strabismus. J AAPOS. 2002:6(3):191-4.

13. Wright KW, Edelman PM, Walonker F, Yiu S. Reliability of fixation preference testing in diagnosing amblyopia. Arch Ophthalmol. 1986;104(4):549-53.

14. Wright KW, Walonker F, Edelman P. 10-Diopter fixation test for amblyopia. Arch Ophthalmol. 1981;99(7):1242-6

15. Friedman DS, Katz J, Repka MX, Giordano L, Ibrionke J, Hawse P, et al. Lack of concordance between fixation preference and HOTV optotype visual acuity in preschool children: the Baltimore Pediatric Eye Disease Study. Ophthalmology. 2008;115(10):1796-9.

16. Cotter SA, Tarczy-Hornoch K, Song E, Lin J, Borchert M, Azen SP, Varma R; Multi-Ethnic Pediatric Eye Disease Study. Fixation preference and visual acuity testing in a population-based cohort of preschool children with amblyopia risk factors. Ophthalmology. 2008;116(1):145-53.Erratum in: Ophthalmology. 2009;116(2):174.

17. Hakim OM. Association between fixation preference testing and strabismic pseudoamblyopia. J Pediatr Ophthalmol Strabismus. 2007;44(3):174-7.

18. Laws D, Noonan CP, Ward A, Chandna A. Binocular fixation pattern and visual acuity in children with strabismic amblyopia. J Pediatr Ophthalmol Strabismus. 2000;37(1):24-8.

19. Zipf RF. Binocular fixation pattern. Arch Ophthalmol. 1976;94(3):401-5.

20. Atilla H, Oral D, Coskun S, Erkam N. Poor correlation between "fix-follow-maintain" monocular/binocular fixation pattern evaluation and presence of functional amblyopia. Binocul Vis Strabismus Q. 2001;16(2):85-90

21. Procianoy L, Procianoy E. The accuracy of binocular fixation preference for diagnosing strabismic amblyopia. J AAPOS. 2010;14(3):205-10. Comment in: J AAPOS. 2010;14(3):201-12

22. Vuillerme N, Isableu B, Nougier V. Attentional demands associated with the use of a light fingertip touch for postural control during quiet standing. Exp Brain Res. 2006; 169(2):232-6. 